\title{
An in vitro study on cleaning efficiency of Mtwo and BioRaCe rotary nickel-titanium instruments
}

\author{
Saeid Zamiran ${ }^{1^{*}}$, Maryam Zare Jahromi ${ }^{2}$, Mohammadhossein Fathi ${ }^{3}$, Ghazaleh Moghaddam ${ }^{4}$ \\ ${ }^{1}$ Endodontist, Private Practice, Teheran, Iran; ${ }^{*}$ Corresponding Author: saeedzamiran@yahoo.com \\ ${ }^{2}$ Department of Endodontics, School of Dentistry, Islamic Azad University, Khorasgan (Isfahan) Branch, Isfahan, Iran \\ ${ }^{3}$ Department of Material Engineering, Isfahan University of Technology, Isfahan, Iran \\ ${ }^{4}$ Department of Drug and Food Control, Faculty of Pharmacy, Tehran University of Medical Sciences, Tehran, Iran
}

Received 19 October 2013; revised 25 November 2013; accepted 3 December 2013

Copyright (C) 2013 Saeid Zamiran et al. This is an open access article distributed under the Creative Commons Attribution License, which permits unrestricted use, distribution, and reproduction in any medium, provided the original work is properly cited.

\begin{abstract}
Complete removal of smear layer and debris during the process of root canal therapy is of paramount importance in order to achieve the objectives of endodontic treatment. The aim of this study was to compare the amount of extruded debris and smear layer using two widespread rotary nickel-titanium instruments. Fifty eight freshly extracted human mandibular first molar mesial canals were randomly assigned into two groups. The root canals were instrumented using Mtwo (VDW, Munich, Germany), and BioRaCe (FKG Dentaire, LaChauxde-Fonds, Switzerland) NiTi instruments. Debris and smear layer from the apical thirds part during instrumentation were evaluated by scanning electron microscope. Then, the scores of remaining debris and smear layer were calculated for each group and compared. Data were then statistically analyzed using Mann-Whitney $U$ test. There were significant differences in the amount of smear layer among two groups ( $P$ $<0.05$ ). The greatest amount of apical debris was extruded by the BioRaCe group and the least by the Mtwo group. The results of the present study showed that, although both instrumentation techniques apically extruded debris and smear layer through the apical foramen, the Mtwo instruments induced less extruded debris and smear layer than the BioRaCe rotary systems.
\end{abstract}

Keywords: BioRaCe; Debris; Mtwo; SEM; Smear Layer

\section{INTRODUCTION}

Recent advances in the field of endodontics have led to the use of nickel titanium (NiTi) rotary instruments in dental practice. The fundamental aim of endodontic treatment is to prevent or cure apical periodontitis. One of the main objectives of root canal preparation is to shape and clean the root canal system effectively whilst maintaining the original configuration without creating any iatrogenic events $[1,2]$. The choice of instruments and systems is a reflection of the different thoughts regarding taper of instrument and taper of the prepared canal. The unique property of super elasticity may allow NiTi files to be placed in curved canals with less lateral force exerted [3-5].

Smear layer differs from the dusty pattern of superficial debris in that it is a layer of muddy material, composed of an amorphous layer of organic-inorganic debris, and bacteria, which is compacted against the dentine walls due to the rasping action of endodontic instruments $[6,7]$. Cleaning is necessary to remove all the pulp tissue, necrotic debris, microorganisms and the infected layer of dentine from the canal walls, whilst shaping involves the enlargement of the canal system to facilitate the placement of a root filling [8].

Traditional cleaning and shaping techniques employing hand instruments have a variety of steps, depend on the clinician's skill, and are often complex [5]. Recently canal preparation is less arduous and more standardized by using NiTi in instrument manufacture and the introduction of high torque rotary handpieces. Rotary instrumentation with NiTi files results in a more uniform preparation with regard to taper and can enhance obturation by providing resistance $[9,10]$.

Currently, the Mtwo rotary nickel-titanium instruments have been utilized widely especially in Iran. The basic series (standard set) of Mtwo rotary files includes four instruments with variable tip sizes ranging from no. 10 to no. 25 , tapers ranging from 0.04 to $0.06-0.07$ and two lengths: 21 and $25 \mathrm{~mm}$. Also file tips ranging in size 
from 30, 35, 40 and tapers of $0.5,0.4$ and 0.7 are available. More recently, BioRaCe was introduced into the market as a new rotary system that is a modification of the RaCe (FKG Dentaire). According to the manufacturer, BioRaCe instruments present the same physical characteristics as $\mathrm{RaCe}$ instruments; BioRaCe differs from the well known RaCe instruments in regard to instruments sizes, tapers and sequence. The major goal of BioRaCe is to achieve apical preparation sizes that are scientifically proven to effectively disinfect the canal [11]. Therefore, based on the related description, the aim of this investigation was to compare the cleaning efficiency (residual debris, and the smear layer) after preparation of severely curved root canals with Mtwo, and BioRaCe files.

\section{MATERIAL AND METHOD}

\subsection{Sample Preparation}

In the present study, 58 freshly extracted human mandibular first molar mesial canals were used including 28 and 30 samples for BioRaCe and Mtwo respectively. Teeth with calcification and open apices were excluded. The used method was the Hankins and El Deeb 1966 [12] preparing a root canal which involved measuring the length of the canal from a radiograph and shaping it from the apical constriction to the orifice. All canals were sequentially prepared at least from size of 10 or 15 . All teeth were analyzed with digital radiographs (RVG, CygnusRay MPS, Cygnus Technologies LLC, USA) in the buccal and proximal directions to check for a single canal. The experimental teeth were washed by $2.5 \%$ sodium hypochlorite and stored in $0.9 \%$ formalin solution before experimentation. All chemicals were purchased from Merck (Darmstadt, Germany). The degree of curvature was $10^{\circ}-20^{\circ}$. The diameter and the taper of all canals were equivalent to an ISO standard size 10 or 15 root canal instrument and the teeth were shortened so that all samples were 19-mm long; then the prepared samples were cleared with doubled de-ionized water and were irrigated with $1 \mathrm{~mL}$ of $5 \% \mathrm{NaOCl}$ followed by 0.5 $\mathrm{mL}$ of $10 \%$ EDTA, respectively.

\subsection{Instrumentation and SEM Examination}

Studied instruments including Mtwo (VDW, Munich, Germany), and BioRaCe (FKG Dentaire, La-Chaux-deFonds, Switzerland) were also utilized according to the manufacturer's instructions. Instruments were withdrawn when resistance was felt and changed for the next instrument. Mtwo instruments were set into permanent rotation with the torque-limited rotation handpiece, at a maximum speed of $3000 \mathrm{rpm}$. According to the manufacturer, the Mtwo instruments should be used in a single length technique with a gentle in-and-out motion. In ad- dition, all files of the instrumentation sequence were used to the full working length of the root canal. Several rotary instruments were used: Mtwo 4\% taper, size 10; Mtwo 5\% taper, size 15; Mtwo 6\% taper, size 20; Mtwo $6 \%$ taper. Additionally, \#15 K-file (FKG) was used for the initial assessment of the canal space and also in a watch-winding motion to assure the presence of a gliding path for each next Mtwo instrument. In BioRaCe group the canals were instrumented at a speed of $500 \mathrm{rpm}$. The instrument was used in a 16:1 gear reduction handpiece powered by a torque-controlled electric motor (X-Smart; Dentsply Mail lefer). The BioRaCe instruments were used with gentle strokes based on the recommendations of the manufacturer. BR0 ( $8 \%$ taper, size 25$)$ was only used in the coronal third. BR1 (5\% taper, size 15), BR2 ( $4 \%$ taper, size 25$)$, BR3 ( $6 \%$ taper, size 25$)$, BR4 (4\% taper, size 35), and BR5 ( $4 \%$ taper, size 40 ) were used in the full working length of the canal. After that each sample was split into two halves with a stainless steel chisel. The section with the most visible part of the apical was conserved and fixed in $4 \%$ glutaraldehyde in $0.2 \mathrm{M}$ sodium cacodylate buffer solution at $4^{\circ} \mathrm{C}$, dehydrated in graded concentration alcohol, dried with a critical point drier (E 3000; Polaron, West Sussex, UK) and then gold sputtered (Sputter Coater; SPI, Toronto, Canada) and observed with SEM (Philips-Germany). After a general survey of the canal wall from the apical part, SEM photomicrographs were obtained at a standard magnification of $\times 750$ at each apical thirds part. The images were saved digitally with specific software and scored based on in a double-blind manner by two trained operators. Separate evaluations were recorded for debris and smear layer. By regarding the report of Schäfer-Schlingmann [13] the cleanliness of each root canal was evaluated in apical thirds areas by means of a numerical evaluation scale. Two endodontists were trained using the mentioned scoring system and calibrated. The inter-examiner reliability tests showed a high consistency between the both of them.

\subsection{Data Analysis}

A database was designed using Microsoft Excel, and data were analyzed using SPSS release 16.0 software (SPSS, Chicago, IL, USA). Briefly, the statistical comparisons between the instruments were computed using the Mann-Whitney $U$ test and the significance was declared at $\mathrm{P}<0.05$.

\section{RESULT AND DISCUSSION}

Following evaluation with SEM at the magnifications of $\times 750$, it was demonstrated that at the apical thirds canal levels, there was significantly cleaner appearance in Mtwo group compared to BioRaCe group in smear layers. 
Similarly, there was also the same manner in the canal cleanliness between the apical levels of these groups in debris which the statistical issues did not reveal any significant differences (Figure 1).

The mean extruded debris, smear layer values and standard deviation for each group are presented in Table 1. The results indicated that both the instruments tested caused measurable apical smear layer and also debris extrusion. Based on the calculation the mean scores of smear layer were $4.40 \pm 0.49$ and $4.85 \pm 0.35$ in Mtwo and BioRaCe respectively. As it was mentioned before a statistically significant difference was found between the Mtwo and BioRaCe in smear layer $(\mathrm{P}<0.05)$. Although the apical thirds of studied samples did not show any significant differences between these groups in debris scores $(\mathrm{P}>0.05)$, lower amount of debris was recorded by Mtwo too.

The frequencies of scores were shown in Figure 2. As can be seen, related scores of BioRaCe in different samples illustrated more variation in both debries and smear layer. However Mtwo scores stayed stable particularly in smear layer.

The main aim of endodontic treatment is the root canal shaping and an effective cleaning in order to remove bacteria from the canal system and prevent the occurrence of apical periodontitis $[14,15]$. Adequate choice of instruments and preparation techniques dramatically reduce the number of bacteria in the canal system prior to obturation [16]. Consistent success in endodontics requires high technical skill in order to achieve a biological goal. It is well established that in order to remove enough microbes from the root canal to ensure predictable success, the apical third of the canal must be instrumented to certain minimum sizes. In the present study, both assessed instruments apically extruded debris and smear layer through the apical foramen. Apical part was selected in this research since based on the previous reports the amount of smear layer and also debris were greater in the apical thirds than in the coronal and middle of the root $[6,7]$. SEM analysis is seemingly adequate for the studies on the cleaning efficacy of different instruments and substances [17]. There are, however, some minor differences such as magnification, area selection, transparent grid assessment and scoring systems that exist in the application of this technique [18]. Mtwo instrument have been available since 2005 . It has an increasing pitch from tip to shaft to eliminate screwing and binding in continuous rotation. Also the transportation of debris towards the apex is decreased [19]. On the other side, the BioRaCe sequence is unique; it has been especially designed to achieve the required apical sizes without the need for additional step and additional files. If used according to instructions, most canals can be effectively cleaned with five NiTi files. Thus with the use of the unique BioRaCe system, the biologic aim of root canal treatment is achieved without compromising efficiency.

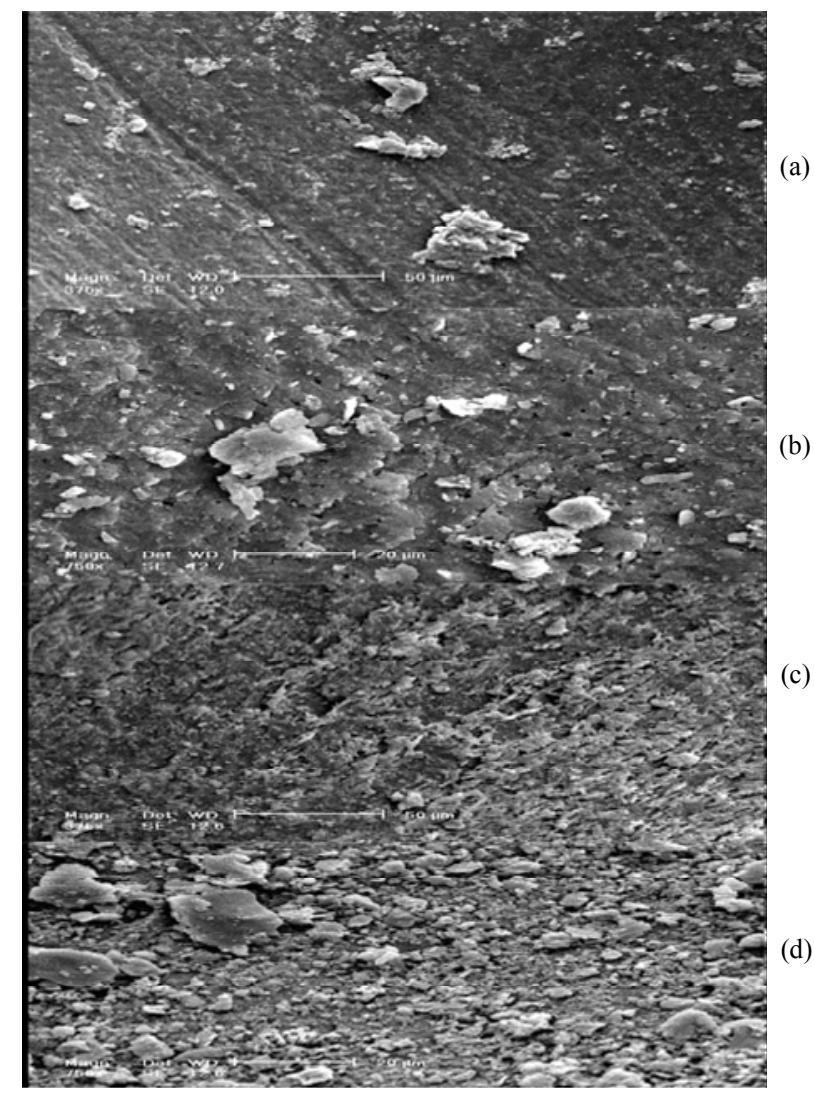

Figure 1. SEM photomicrographs of debris in (a) Mtwo, (b) BioRaCe and smear layer in (c) Mtwo, (d) BioRaCe apical third area. Original magnification $\times 750$.

Table 1. Scores of smear layer and debris in Mtwo and BioRaCe.

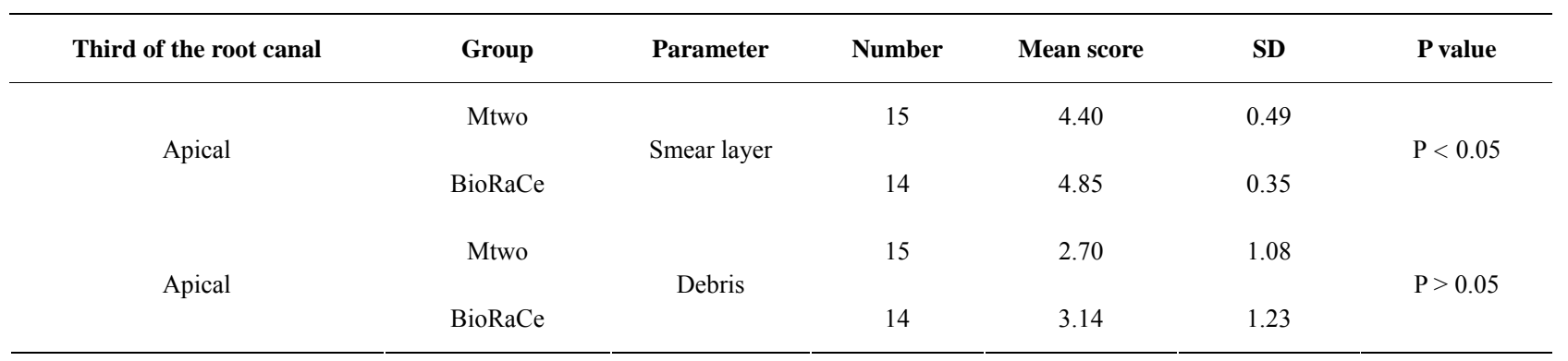




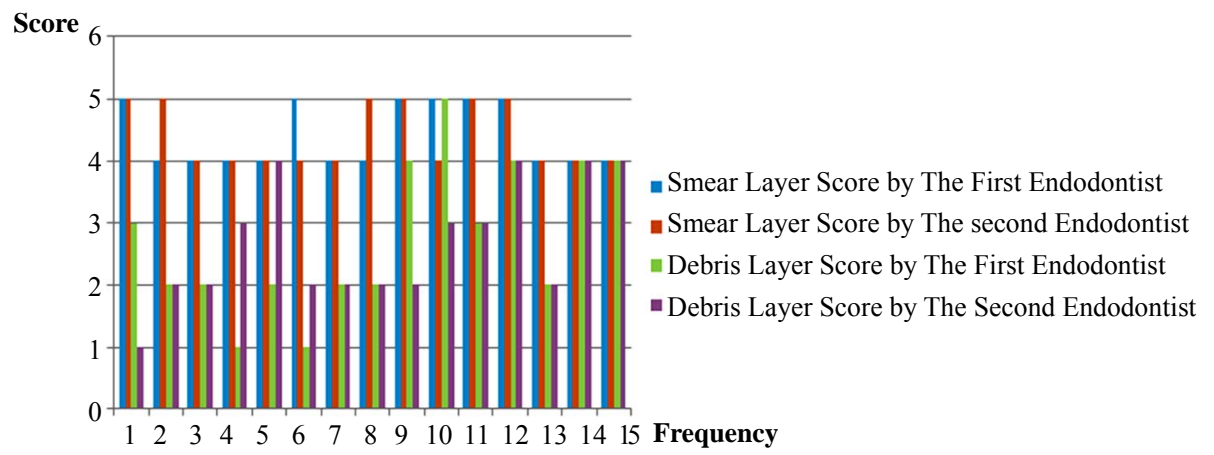

(a)

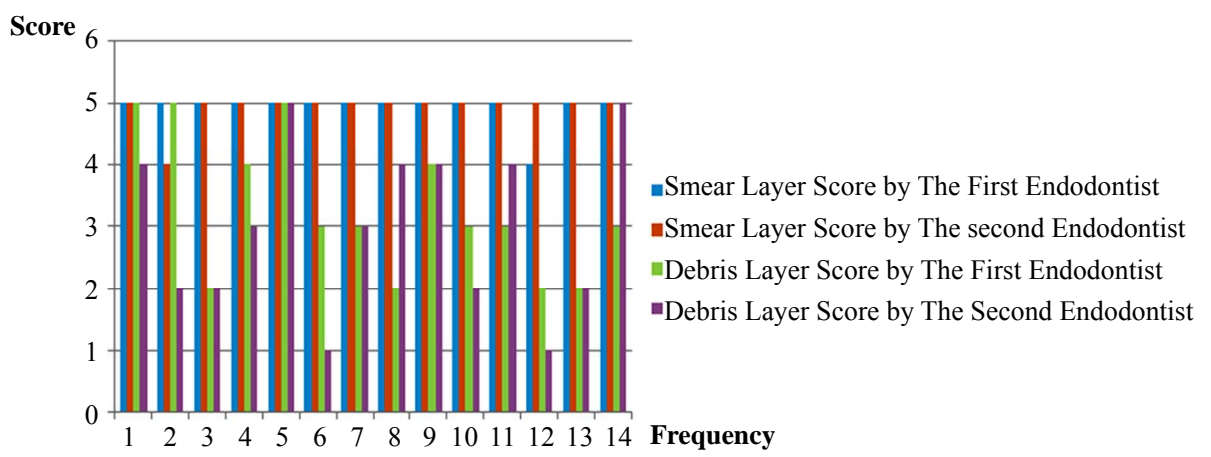

(b)

Figure 2. Comparative illustration of smear layer and debris scores for (a) Mtwo and (b) BioRaCe group. Different colors represent different scores as presented in the legends.

It is expected that application of BioRaCe, provides biologically appropriate diameter in the apical portion of the canal and thus a lower number of residual bacteria to ensure safe and effective obturation [20].

Within the parameters of this study, the use of Mtwo files resulted in significantly less smear layer compared with canal preparation of BioRaCe instruments, whereas in terms of debris no statistically significant differences were apparent.

Shafer et al. claimed that Mtwo files maintained the original curvature significantly better than the K3 and RaCe systems [21]. Additionally, in another study, Mtwo instruments respected original canal curvature well and prepared curved canals rapidly without substantial change in working length. Therefore, the results indicate that Mtwo instruments were safe to use [21]. Moreover, perfect centring ability was detected with removal of material uniformly both on the outer and inner side of the curvature [21]. In contrast, in Tasdemir et al. paper the BioRaCe instruments induced less extruded debris than the Mtwo rotary systems [11]. The reason may be attributed to its non-convex triangular cross-sectional design.

To compare with other similar researches, the use of Mtwo instrument in Foschi et al. research produced a clean and debris-free dentine surface in the coronal and middle thirds; on the contrary, these instruments were unable to produce a dentine surface free from smear layer and debris in the apical third. Deep grooves presence and depression on dentine walls in the apical third may well explain the presence of less instrumented areas [6].

The shape of Mtwo instruments with two blades and a large groove between them seems to reduce the core diameter and increase the flexibility; thus it is anticipated that, due to the active sharp angle of the blade, the resistance of the instrument could not be affected (Figure 3 ). The blade angle is almost vertical and the helical pitches increases from tip to handle. These characteristics are claimed to decline debris accumulation and to gain a more effective cutting ability [22]. It must be emphasized that another advantage of this instrument is the instrumentation time which is substantially faster than all other rotary nickel-titanium instruments tested under identical experimental conditions [23].

Taşdemir et al. studied on the comparison of apically extruded debris using three rotary nickel-titanium instruments including BioRaCe, Mtwo and ProTaper Universal. There were significant differences in the amount of debris extruded among three groups. The greatest amount of apical debris was extruded by the ProTaper Universal group and the least by the BioRaCe group. The achieved results of our study were not approved by Taşdemir study which the BioRaCe instruments induced less extruded debris than the ProTaper Universal and Mtwo 


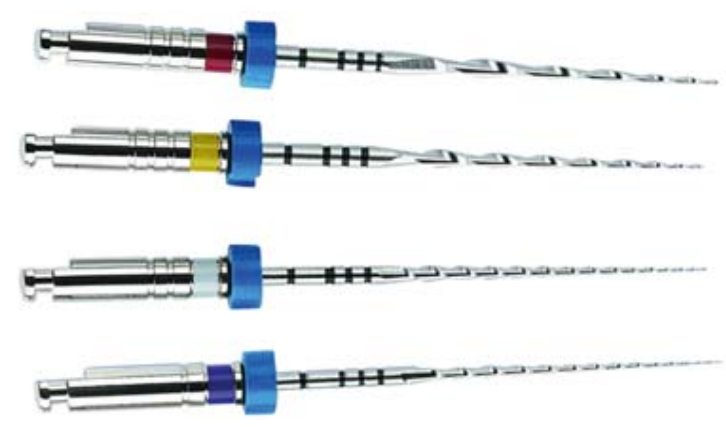

(a)
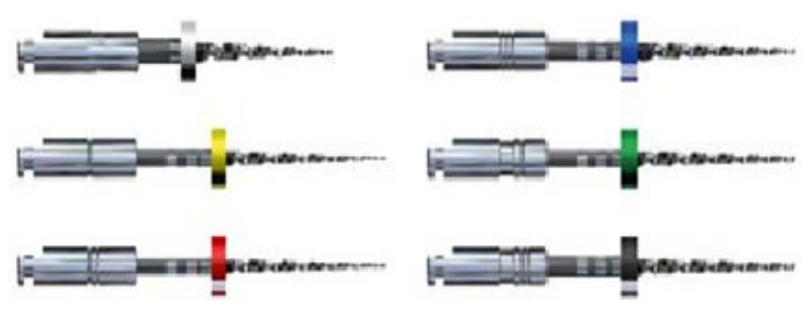

(b)

Figure 3. The appearance of (a) Mtwo and (b) BioRaCe nickeltitanium instruments.

rotary systems [11].

The variation between the results from different studies is logical due to varying experimental set ups and investigated elements. Consequently, the choice of a specific rotary system needs consideration of the combined evaluation various parameters. Also BioRace as an update system is the most promising because studies about it still need to be accomplished.

\section{CONCLUSION}

Since root canal infection is the cause of apical periodontitis, the biological aim of endodontic treatment is the prevention or elimination of root canal microbes. Based on the major objective of treatment, it could be concluded that Mtwo rotary instrument removed smear layer effectively in the third apical part of the canal, but Bio$\mathrm{RaCe}$ showed more debris and smear layer.

\section{ACKNOWLEDGEMENTS}

This study was a part of a Ph.D. thesis and a project has been funded and supported by Isfahan University of Technology, Isfahan-Iran and also Islamic Azad University-Khorasgan, Isfahan-Iran.

\section{REFERENCES}

[1] Sanghvi, Z. and Mistry, K. (2011) Design features of rotary instruments in endodontics. Journal of Ahmedabad Dental College and Hospital, 2, 6.

[2] Gambarini, G. and Laszkiewicz, J. (2002) A scanning electron microscopic study of debris and smear layer remaining following use of GT rotary instruments. Interna- tional Endodontic Journal, 35, 422-427. http://dx.doi.org/10.1046/j.1365-2591.2002.00495.x

[3] Bergmans, L., et al. (2001) Mechanical root canal preparation with NiTi rotary instruments: Rationale, performance and safety. American Journal of Dentistry, 14, 324333.

[4] Gharai, S.R., et al. (2005) Comparison of generated forces and apical microleakage using nickel-titanium and stainless steel finger spreaders in curved canals. Journal of Endodontics, 31, 198-200. http://dx.doi.org/10.1097/01.don.0000137648.77797.ea

[5] Young, G., Parashos, P. and Messer, H. (2007) The principles of techniques for cleaning root canals. Australian Dental Journal, 52, S52-S63.

[6] Foschi, F., et al. (2004) SEM evaluation of canal wall dentine following use of Mtwo and ProTaper NiTi rotary instruments. International Endodontic Journal, 37, 832839. http://dx.doi.org/10.1111/j.1365-2591.2004.00887.x

[7] Drukteinis, S. and Balciuniene, I. (2006) A scanning electron microscopic study of debris and smear layer remaining following use of AET instruments and K-flexofiles. Stomatologija, 8, 70-75.

[8] Alodeh, M., Doller, R. and Dummer, P. (1989) Shaping of simulated root canals in resin blocks using the step-back technique with K-files manupulated in a simple in/out filing motion. International Endodontic Journal, 22, 107117. http://dx.doi.org/10.1111/j.1365-2591.1989.tb00908.x

[9] Von Fraunhofer, J., et al. (2000) The effect of root canal preparation on microleakage within endodontically treated teeth: An in vitro study. International Endodontic Journal, 33, 355-360. http://dx.doi.org/10.1046/j.1365-2591.2000.00318.x

[10] Fayyad, D.M. and Elhakim Elgendy, A.A. (2011) Cutting efficiency of twisted versus machined nickel-titanium endodontic files. Journal of Endodontics, 37, 1143-1146. http://dx.doi.org/10.1016/j.joen.2011.03.036

[11] Tasdemir, T., et al. (2010) An $<\mathrm{i}>$ in vitro $</ \mathrm{i}>$ comparison of apically extruded debris using three rotary nickeltitanium instruments. Journal of Dental Sciences, 5, 121125. http://dx.doi.org/10.1016/S1991-7902(10)60017-7

[12] Hankins, P.J. and ElDeeb, M.E. (1996) An evaluation of the canal master, balanced-force, and step-back techniques. Journal of Endodontics, 22, 123-130. http://dx.doi.org/10.1016/S0099-2399(96)80288-3

[13] Schäfer, E. and Schlingemann, R. (2003) Efficiency of rotary nickel-titanium K3 instruments compared with stainless steel hand K-Flexofile. Part 2. Cleaning effectiveness and shaping ability in severely curved root canals of extracted teeth. International Endodontic Journal, 36, 208-217. http://dx.doi.org/10.1046/j.1365-2591.2003.00644.x

[14] Wu, M.K., Dummer, P. and Wesselink, P. (2006) Consequences of and strategies to deal with residual posttreatment root canal infection. International Endodontic Journal, 39, 343-356. http://dx.doi.org/10.1111/j.1365-2591.2006.01092.x

[15] Wu, M., Sluis, L. and Wesselink, P. (2003) The capability of two hand instrumentation techniques to remove the 
inner layer of dentine in oval canals. International Endodontic Journal, 36, 218-224.

http://dx.doi.org/10.1046/j.1365-2591.2003.00646.x

[16] Siqueira, J. (2001) Aetiology of root canal treatment failure: Why well-treated teeth can fail. International Endodontic Journal, 34, 1-10.

http://dx.doi.org/10.1046/j.1365-2591.2001.00396.x

[17] Spangberg, L.S. and Haapasalo, M. (2002) Rationale and efficacy of root canal medicaments and root filling materials with emphasis on treatment outcome. Endodontic Topics, 2, 35-58.

http://dx.doi.org/10.1034/j.1601-1546.2002.20104.x

[18] Jeon, I.-S., et al. (2003) Smear layer production by 3 rotary reamers with different cutting blade designs in straight root canals: A scanning electron microscopic study. Oral Surgery, Oral Medicine, Oral Pathology, Oral Radiology, and Endodontology, 96, 601-607. http://dx.doi.org/10.1016/S1079-2104(03)00303-2

[19] Vaudt, J., Bitter, K. and Kielbassa, A.M. (2007) Evaluation of rotary root canal instruments in vitro: A review. Endodontics, 1, 189-203.

[20] Debelian, G. (2011) BioRaCe NiTi system: Biologically desirable apical sizes: Safely and efficiently. Stomatološki Glasnik Srbije, 58, 44-50.

http://dx.doi.org/10.2298/SGS1101044D

[21] Schäfer, E., Erler, M. and Dammaschke, T. (2006) Comparative study on the shaping ability and cleaning efficiency of rotary Mtwo instruments. Part 2. Cleaning effectiveness and shaping ability in severely curved root canals of extracted teeth. International Endodontic Journal, 39, 203-212.

http://dx.doi.org/10.1111/j.1365-2591.2006.01075.x

[22] Veltri, M., et al. (2005) A comparative study of Endoflare-Hero Shaper and Mtwo NiTi instruments in the preparation of curved root canals. International Endodontic Journal, 38, 610-616. http://dx.doi.org/10.1111/j.1365-2591.2005.00989.x

[23] Schäfer, E., Erler, M. and Dammaschke, T. (2006) Comparative study on the shaping ability and cleaning efficiency of rotary Mtwo instruments. Part 1. Shaping ability in simulated curved canals. International Endodontic Journal, 39, 196-202.

http://dx.doi.org/10.1111/j.1365-2591.2006.01074.x 